\title{
Kinetic alterations of collagen and elastic fibres and their association with cardiac function in acute myocardial infarction
}

\author{
YUEXIN YU ${ }^{1}$, GUOTIAN YIN ${ }^{2}$, SHISAN BAO ${ }^{3}$ and ZHIKUN GUO ${ }^{1}$ \\ ${ }^{1}$ Henan Key Laboratory of Medical Tissue Regeneration, Xinxiang Medical University, Xinxiang, \\ Henan 453003; ${ }^{2}$ Department of Cardiology, Third Affiliated Hospital of Xinxiang Medical University, \\ Xinxiang, Henan 453000, P.R. China; ${ }^{3}$ Discipline of Pathology, School of Medical Sciences and Bosch Institute, \\ Sydney Medical School, The University of Sydney, Sydney, New South Wales 2006, Australia
}

Received March 17, 2017; Accepted September 14, 2017

DOI: $10.3892 / \mathrm{mmr} .2017 .8347$

\begin{abstract}
The present study aimed to investigate kinetic alterations of collagen and elastic fibres and their association with cardiac function in the acute myocardial infarction (AMI) heart. AMI was generated in Sprague-Dawley rats by ligation of the left anterior descending coronary artery. Cardiac function was determined using B-ultrasonography, AMI was verified using histopathology. The kinetics of collagen type I/III and elastic fibre were evaluated using immunohistochemistry and western blotting at 1 week $(1 \mathrm{w}), 2$ weeks $(2 \mathrm{w}), 3$ weeks $(3 \mathrm{w})$ and 4 weeks $(4 \mathrm{w})$ post-AMI. Cardiac function was decreased by $78,70,50$ and $38 \%$ at weeks $1,2,3$ or 4 post-AMI, respectively, compared with the normal heart. Elastic fibre was decreased gradually, demonstrating alterations of 2, 77, 86 or $97 \%$ reduction, respectively, at weeks $1,2,3$ or 4 in the AMI heart. Collagen I fibre was increased 1.4-, 1.5-, 2.9- or 3.9-fold at weeks 1, 2, 3 or 4 respectively, compared with the normal heart. Similarly, collagen III was increased 1.2-, 1.7-, 2.8- or 3.9-fold, following AMI. Kinetics of increased collagen I/III, in combination with decreased elastic fibre in infarcted area following AMI, provided evidence that compromised cardiac function following AMI was due to graduate wound healing/scar formation in the infarcted zone, increased stiffness and reduced flexibility of the heart.
\end{abstract}

\section{Introduction}

Acute myocardial infarction (AMI) remains of primary concern, resulting in a huge physical and economic burden around the world, despite decades of extensive research. Heart failure gradually develops due to loss of cardiomyocytes, which

Correspondence to: Dr Zhikun Guo, Henan Key Laboratory of Medical Tissue Regeneration, Xinxiang Medical University, 601 Jinsui Road, Xinxiang, Henan 453003, P.R. China

E-mail: gzk@xxmu.edu.cn

Key words: elastic fibre, collagen I/III, acute myocardial infarction, cardiac function are replaced with scar tissue post-AMI. It has been reported that there are other intercellular alterations, including collagen and elastic fibres in the infarcted region (1-3). At present, research interests focus on alterations of cardiomyocytes and the neogenesis of capillaries following AMI $(4,5)$.

The extracellular matrix is an important component of the heart, and includes collagen fibre, reticular fibre and elastic fibres with distinctive functions. These fibres constitute a complex network of cardiac fibre stents for maintaining heart form and function (6). Myocardial collagen fibre, elastic fibre and reticular fibre increase gradually from embryonic stage to birth, and then to adulthood. The fastest fibre growth is from childhood to youth, and tends to be stable following the onset of adulthood (7). Elastic fibres exhibit an indispensable role in maintaining cardiac morphology and function, although the content of elastic fibre is relatively lower compared with collagen fibre in the heart. In the events of disproportional distribution between the collagen fibre and elastic fibre, normal heart function will be compromised, resulting in electrophysiological systolic and diastolic functional alterations, which may eventually progress to heart failure (8). Intercellular collagen and elastic fibre will have different degrees of deposition subject to damage or reconstruction following AMI.

However, the kinetic alterations of interstitial collagen and elastic fibres during AMI, and in particular, their association with cardiac function, remain to be fully elucidated $(9,10)$. The present study aimed to explore the kinetics of type I/III collagen fibre and elastic fibre in infarcted tissue at different times following AMI and the association with cardiac function. The data may provide a novel insight for myocardial regeneration, which may be of interest to researchers and clinicians.

\section{Materials and methods}

Rat model of AMI. A total of 50 healthy adult Sprague-Dawley rats (age, 4-6 weeks), female only, weighing 230 $20 \mathrm{~g}$, were obtained from the Laboratory Animal Centre, Xinxiang Medical University (Xinxiang, China), animal number: SYXK (YU) 2009-0002. The rats were separately maintained in an animal room between 20 and $23^{\circ} \mathrm{C}$, under a 12-h light/dark cycle, with free access to standard rat feed and ordinary tap water. 
These rats were randomly divided into non-infarcted and infarcted week 1-4 groups $(n=10)$. The experiment strictly adhered to the Guidance for the Care and Use of Laboratory Animals formulated by The Ministry of Science and Technology, China. The present study was approved by the ethics committee of Xinxiang Medical University.

Generation of myocardial infarction: Following weighing, animals were anaesthetised with $10 \%$ chloral hydrate intraperitoneally (i.p. $3 \mathrm{ml} / \mathrm{kg}$ ). The thoracic cavity was exposed following surgical area disinfection with iodine. An artificial breathing machine (Shanghai Biowill Co., Ltd., Shanghai, China) was used for artificial respiration. The left anterior descending coronary artery was ligated following opening of the pericardium, then the chest was closed. A dose of $1.60 \times 10^{7}$ units of penicillin were administered i.p. immediately following surgery, and $0.4 \times 10^{7}$ units of penicillin were administered daily for three days post-operation, following myocardial infarction.

Heart function monitoring and myocardial pathological examination. Intraoperative electrocardiogram (ECG) was used on a BL-420 biological function experimental system, using a small animal ultrasonic instrument MS-250 probe [Winsun (China) Limited, Beijing, China]. Heart function was assessed using the following parameters: left ventricular ejection fraction (LVEF), left ventricular fraction shortening (LVFS), interventricular septal (IVS) thickness, left ventricular internal diameter at end-diastolic (LVIDd), end-systolic (LVIDs) and left ventricular posterior wall (LVPW) thickness.

Immunohistochemistry of type I, III collagen fibre and elastic fibre. The hearts were washed in normal saline and fixed in $4 \%$ paraformaldehyde at $25-28^{\circ} \mathrm{C}$ for $24 \mathrm{~h}$ following sacrificing. The paraffin-embedded blocks $(5 \mu \mathrm{m})$ were sectioned for Masson staining at $25-28^{\circ} \mathrm{C}$ for $20 \mathrm{~min}$ for histopathology with an inverted microscope (TE2000U-PH-E; Leica Microsystems GmbH, Wetzlar, Germany) Immunohistochemistry was then performed as previously described (11). Briefly, paraffin sections were incubated in $0.01 \mathrm{~mol} / 1$ citric acid salt boil buffer for $15 \mathrm{~min}$. After cooling at room temperature, the sections were incubated with $3 \% \mathrm{H}_{2} \mathrm{O}_{2}$ deionized water for $15 \mathrm{~min}$ and washed three times with PBS (5 min/wash), then dewaxed and rehydrated. The sections were incubated with primary antibodies [mouse anti-rat collagen I (Abcam, Cambridge, UK; catalog no. ab90395; 1:1,000) or III (Abcam; catalog no. ab6310; 1:600) or, rabbit polyclonal anti-rat elastin (EterLife, Tianjin, China; catalog no. EL913879; $1: 200)$ overnight at $4^{\circ} \mathrm{C}$. PBS phosphate buffer (dry powder) (catalog no. top0027; Beijing Biotopped Technology Co., Ltd., Beijing, China) was used as an isotype control. Polymer helper was added on the sections for $20 \mathrm{~min}$ at room temperature following PBS washing, then followed by addition of polyperoxidase-anti-mouse/rabbit IgG (ready to use; catalog no. PV-9000; OriGene Technologies, Inc., Beijing, China) for $30 \mathrm{~min}$ at room temperature. DAB was finally added for colour development. The slides were lightly counterstained with haematoxylin at $25-28^{\circ} \mathrm{C}$ for $2 \mathrm{~min}$, and dehydration and transparency-rendering were conducted prior to observation of each section at x100 magnification under an inverted microscope (TE2000U-PH-E; Leica Microsystems $\mathrm{GmbH}$ ).

Immunofluorescence detection of type I, III collagen fibre and elastic fibre in the myocardial infarction zone. Frozen sections $(8 \mu \mathrm{m})$ from AMI and control heart specimens, dried at room temperature, were fixed in cold acetone for $10 \mathrm{~min}$ at $4^{\circ} \mathrm{C}$, washed three times with PBS (5 min/wash), and blocked with normal goat serum (New Zealand origin; $100 \mathrm{ml}$; Thermo Fisher Scientific, Inc., Waltham, MA, USA) at $25-28^{\circ} \mathrm{C}$ for $30 \mathrm{~min}$. The sections were incubated with primary antibodies [mouse anti-rat collagen I (Abcam; catalog no. ab90395; 1:1,000) or III (Abcam; catalog no. ab6310; 1:600) or rabbit polyclonal anti-rat elastin (EterLife; catalog no. EL913879; 1:200) overnight at $4^{\circ} \mathrm{C}$. PBS phosphate buffer (dry powder) (catalog no. top0027; Beijing Biotopped Technology Co., Ltd.) was used as an isotype control. A fluorescein isothiocyanate-conjugated goat anti-rabbit CY3 secary antibody (catalog no. A0516; Beyotime Institute of Biotechnology, Haimen, China; 1:500) was incubated with sections for $1 \mathrm{~h}$ at room temperature. Images were captured under an inverted fluorescence microscope (DMI3000; Leica Microsystems $\mathrm{GmbH}$ ).

Western blotting detection of type I, III collagen fibre and elastic fibre. LV sections of hearts were collected from AMI and normal animals. Total proteins were extracted using a radioimmunoprecipitation assay lysis buffer (Beyotime Institute of Biotechnology), and proteins were quantified using the bicinchoninic acid method. Proteins (30 $\mu \mathrm{g} / \mathrm{lane})$ were separated by $10 \%$ SDS-PAGE. The protein was transferred to a polyvinylidene fluoride membrane and blocked with $5 \%$ skimmed milk for $2 \mathrm{~h}$ at $25-28^{\circ} \mathrm{C}$. Anti-collagen I (1:2,000); anti-collagen III $(1: 4,000)$; or anti-elastin primary antibodies (1:200), as aforementioned, were incubated with the membrane overnight at $4^{\circ} \mathrm{C}$. Horseradish peroxidase-labelled goat anti-mouse secondary antibody (Beyotime Institute of Biotechnology; catalog no. A0216; 1:500) was added for incubation at room temperature for $1 \mathrm{~h}$, followed by addition of an enhanced chemiluminescence luminous fluid (Beyotime Institute of Biotechnology) at room temperature for $3 \mathrm{~min}$. The gel was photographed using a gel imaging system. GAPDH (catalog no. AG019; Beyotime Institute of Biotechnology) was used as a house-keeping reference. The density of the target(s) was determined as the ratio between the target and GAPDH, which was expressed as semi-quantitative.

Statistical analysis. Gel phase system (version 5.3; National Institutes of Health; Bethesda, MD, USA) and Image J (version 2.1.4.7; National Institutes of Health) were used to analyse photographs of western blotting tests and Image-Pro Plus Analysis Systems (version 6.0; Media Cybernetics, Inc., Rockville, MD, USA) for analysis of immunohistochemistry and immunofluorescence, five horizons of clear and non-overlapping tissue in infarcted area were selected for semi-quantitative analysis. The data were analysed by SPSS software, version 19.0 (IBM SPSS, Armonk, NY, USA). One-way analysis of variance followed by Turkey post hoc test were used to assess differences between groups. Data are expressed as the mean \pm standard deviation. $\mathrm{P}<0.05$ was considered to indicate a statistically significant difference. 
A

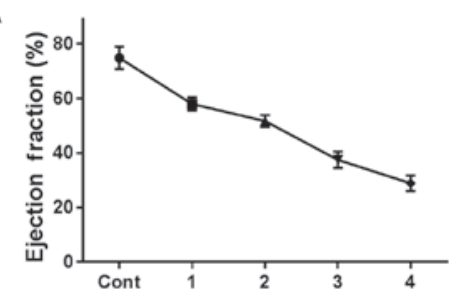

C

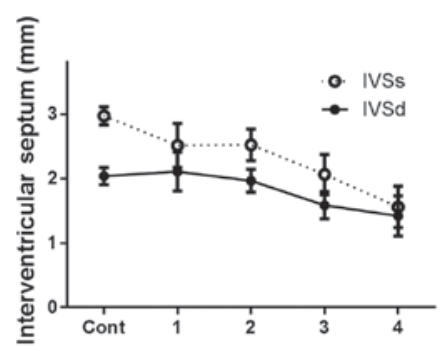

$\mathrm{E}$

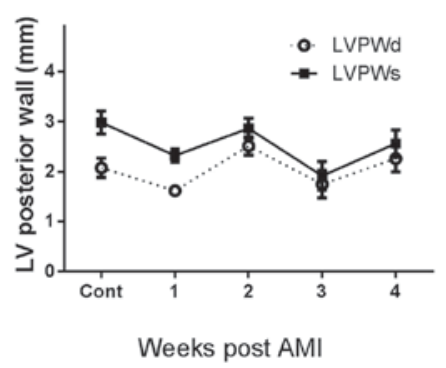

B

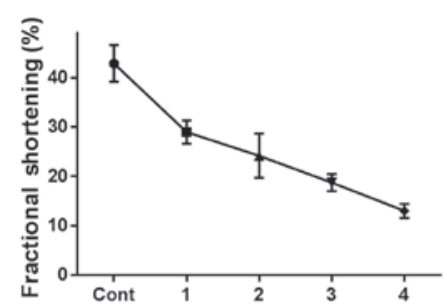

D

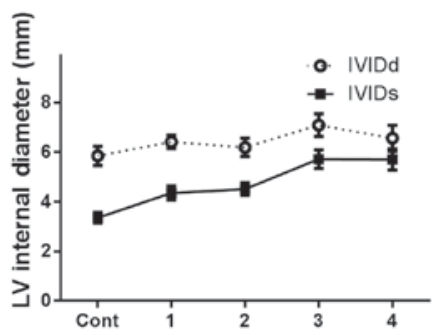

Figure 1. Cardiac function following differing time period post-AMI (weeks). (A) Ejection Fraction (\%), (B) fractional Shortening (\%), (C) interventricular septum (mm), (D) LV internal diameter (mm), (E) LV posterior wall (mm). d, diastolic; s, systolic; AMI, acute myocardial infarction; LV, left ventricular.

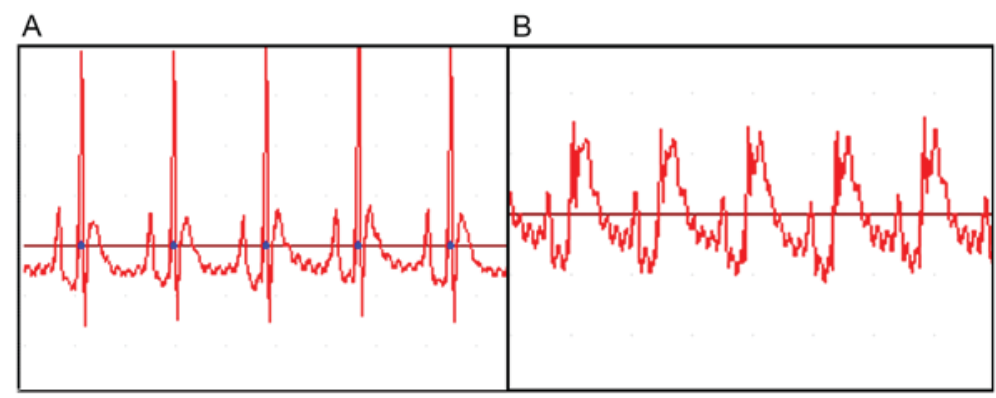

Figure 2. ECG results. An ECG was conducted for heart function monitoring of (A) control and (B) myocardial infarction rats. ECG, electrocardiogram.

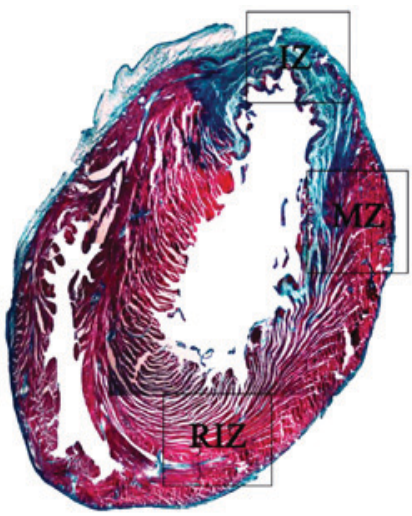

Figure 3. Masson Trichrome staining of heart. Representative image of heart tissue 4 weeks post-acute myocardial infarction, detected by Masson Trichrome staining. IZ, infarct zone; MZ, marginal zone; RIZ, remote infarct zone.

\section{Results}

Cardiac function alterations at different periods following $A M I$.LVEF, LVFS and IVS were gradually decreased, however LVIDs and LVIDd increased with post-AMI time, compared with the normal heart, demonstrating impairment of heart function. In addition, cardiac function gradually decreased with time, particularly 2-3 weeks following infarction. Myocardial infarction models were successfully established and LVPW was not altered (Fig. 1).

Verification of myocardial infarction. Following ligation of the left anterior descending coronary artery, the myocardium below the ligation turned purple or pale, and the left auricle congested heart beat weakened. AMI was diagnosed using an ECG, and the heart rate slowed, with varying degrees of 

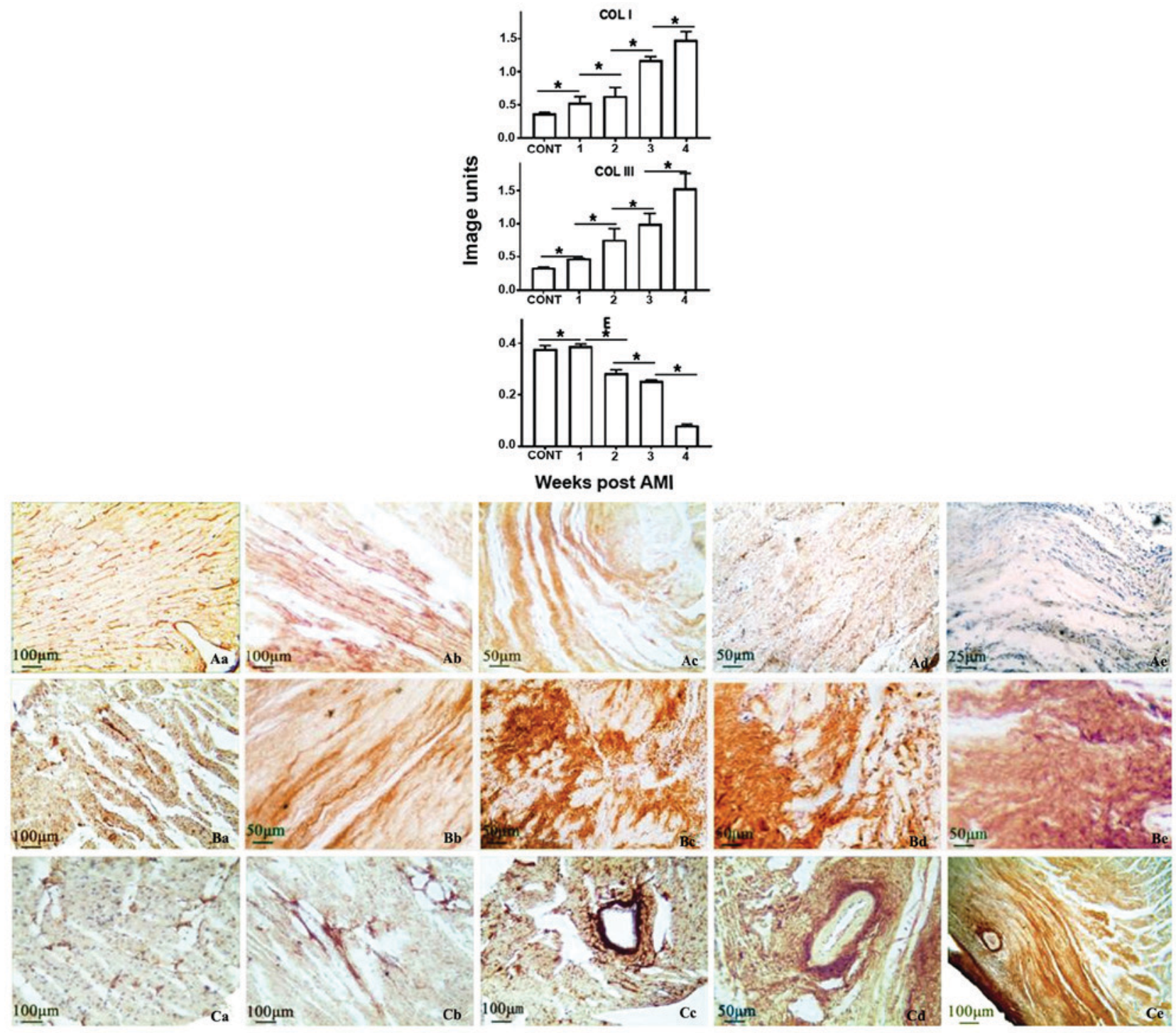

Figure 4. Immunohistochemistry of COL I, III and E fibres. Representative images and semi-quantitative analysis of (A) COL I, (B) COL III and (C) E following AMI (weeks). Subparts a-e indicate the expression of each substance (A), (B) or (C) in the normal group and weeks 1-4 post AMI, respectively. ${ }^{*} \mathrm{P}<0.05$ as indicated. AMI, acute myocardial infarction; COL, collagen; E, elastin.

arrhythmia. II ST segment of the infarction group shifted 20 min following ligation, leading to an ST segment arch upward-push by $0.2 \mathrm{mV}$ (Fig. 2), which demonstrated that that the ligature was successful and the myocardial infarction model had been successfully established.

Histopathological verification of AMI. Paraffin-embedded and Masson stained heart section was evaluated for AMI. Undamaged heart tissue was presented as red, however AMI damaged heart was blue, due to collagen fibre replacement. The front wall of the left ventricle was significantly thinner, infarcted myocardial tissue was replaced by fibrous scar tissue (Fig. 3), confirming the myocardial infarction model.

Immunohistochemistry and immunofluorescence detection of collagen and elastic fibre in the infarcted heart. Using immunohistochemistry, production of collagen I was demonstrated to be gradually increased with AMI time, and revealed a 1.4-, 1.5-, 2.9- or 3.9-fold increase at the weeks $1,2,3$ or 4 , compared with the normal heart, respectively. Similarly, collagen III was increased 1.2-, 1.9-, 2.5- or 3.9-fold, respectively. In contrast, elastin was decreased gradually, and revealed a $2,77,86$ or $97 \%$ reduction, compared with the normal heart, at weeks 1,2, 3 or 4 post-AMI, respectively. Elastic fibre was almost completely replaced by collagen fibre in the infarcted zone, forming scar tissue, at week 4 post-AMI (Figs. 3 and 4). Immunofluorescence was additionally utilised to verify the results obtained from immunohistochemistry analysis. Similar trends were identified in elastin and collagen III [identified in red (CY3)] production, or collagen I [identified in green (FITC)] in the infarcted heart, post-AMI (Fig. 5).

Collagen and elastic fibre protein expression levels are varied in the infarcted heart. Collagen I was increased gradually following increased AMI time, and revealed a 1.5-, 2.0-, 2.5- or 2.6-fold increase at weeks $1,2,3$ or 4, respectively, 

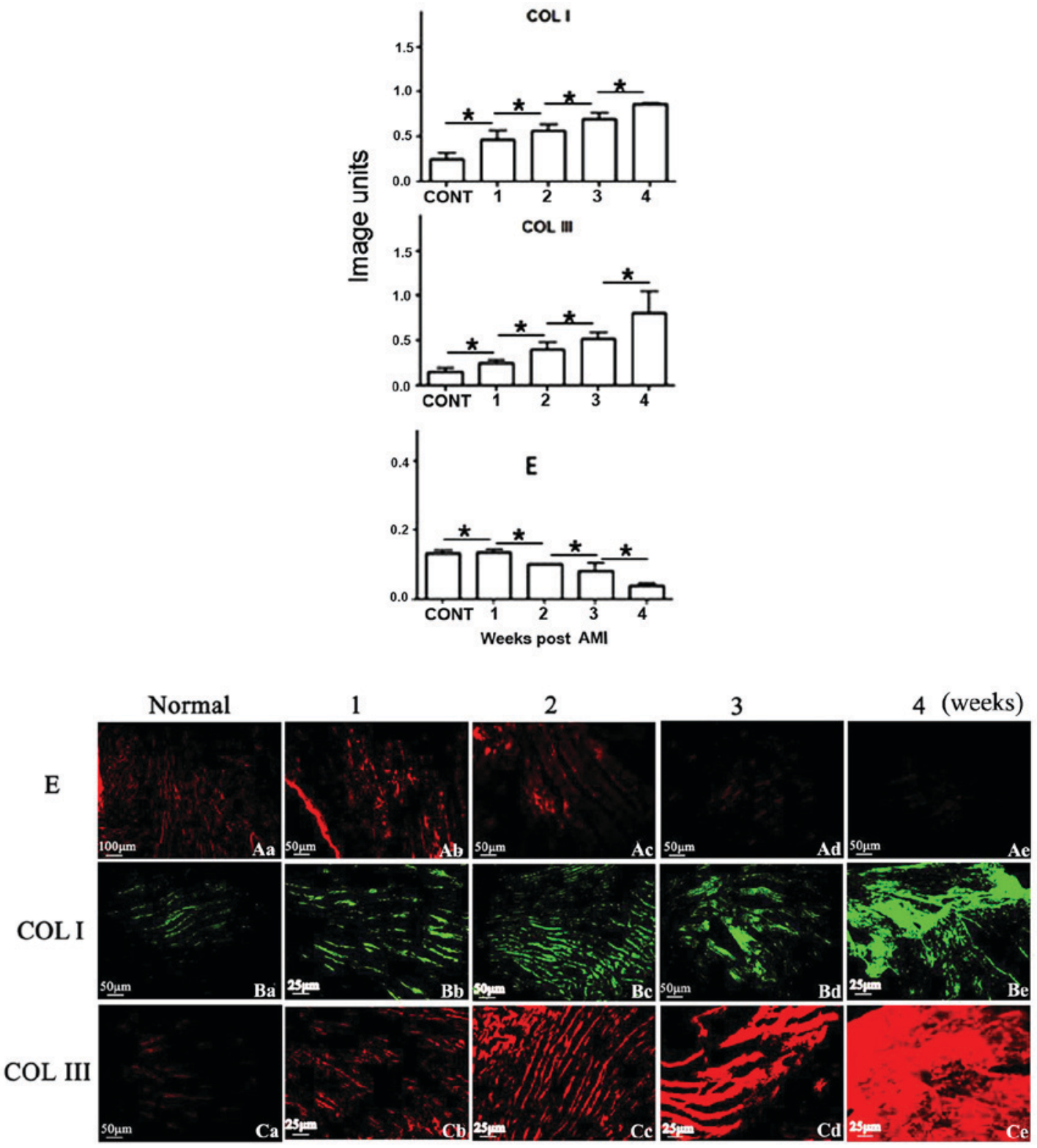

Figure 5. Immunofluorescence analysis of COL I, III and E fibres. Representative images and semi-quantitative analysis of (A) E (B) COL I and (C) COL III following AMI (weeks). Subparts a-e indicate the expression of each substance (A), (B) or (C) in the normal group and weeks 1-4 post AMI, respectively. ${ }^{*} \mathrm{P}<0.05$ as indicated. AMI, acute myocardial infarction; COL, collagen; E, elastin.

compared with the normal heart. Collagen III demonstrated a similar trend, with a 1.0-, 1.3-, 1.5- or 2.5-fold increase at weeks $1,2,3$ or 4 , respectively (Fig. 6). In contrast, elastin was decreased gradually with AMI time, and revealed a 4 , 78,87 or $98 \%$ reduction, compared with the normal heart at weeks 1,2, 3 or 4 , respectively, the comparison between groups was statistically significant $(\mathrm{P}<0.05)$. The results of the western blotting assay were consistent with those obtained via immunohistochemistry and immunofluorescence assays.

\section{Discussion}

The results of the present study demonstrated gradually increased collagen I/III over the four weeks post-AMI, which was consistent with gradually compromised cardiac function. Furthermore, there was an inverse association between increased collagen I/III and decreased elastin in the infarcted heart, which suggested that elastic fibre is critical in maintaining correct cardiac function.

Scar structure is variable at the different periods following myocardial infarction. Following AMI, adjustment of collagen I/III fibre and elastic fibre composition determines cardiac function via ventricle wall compliance and tensile strength (12). An increase in the collagen I/III component augments heart stiffness, which subsequently aggravates left ventricular diastolic and systolic dysfunction $(13,14)$. These previous findings are in accordance with the results of the present study, and collectively suggest that a compromised the function of the heart, resulting in reconstruction of heart configuration, leads to cardiac dysfunction and even heart failure.

Due to the uneven distribution of elastic fibre in the myocardium, and its relatively small content compared with collagen fibre, the literature regarding its specific functions is sparse $(12,15)$. However, it has previously been demonstrated 


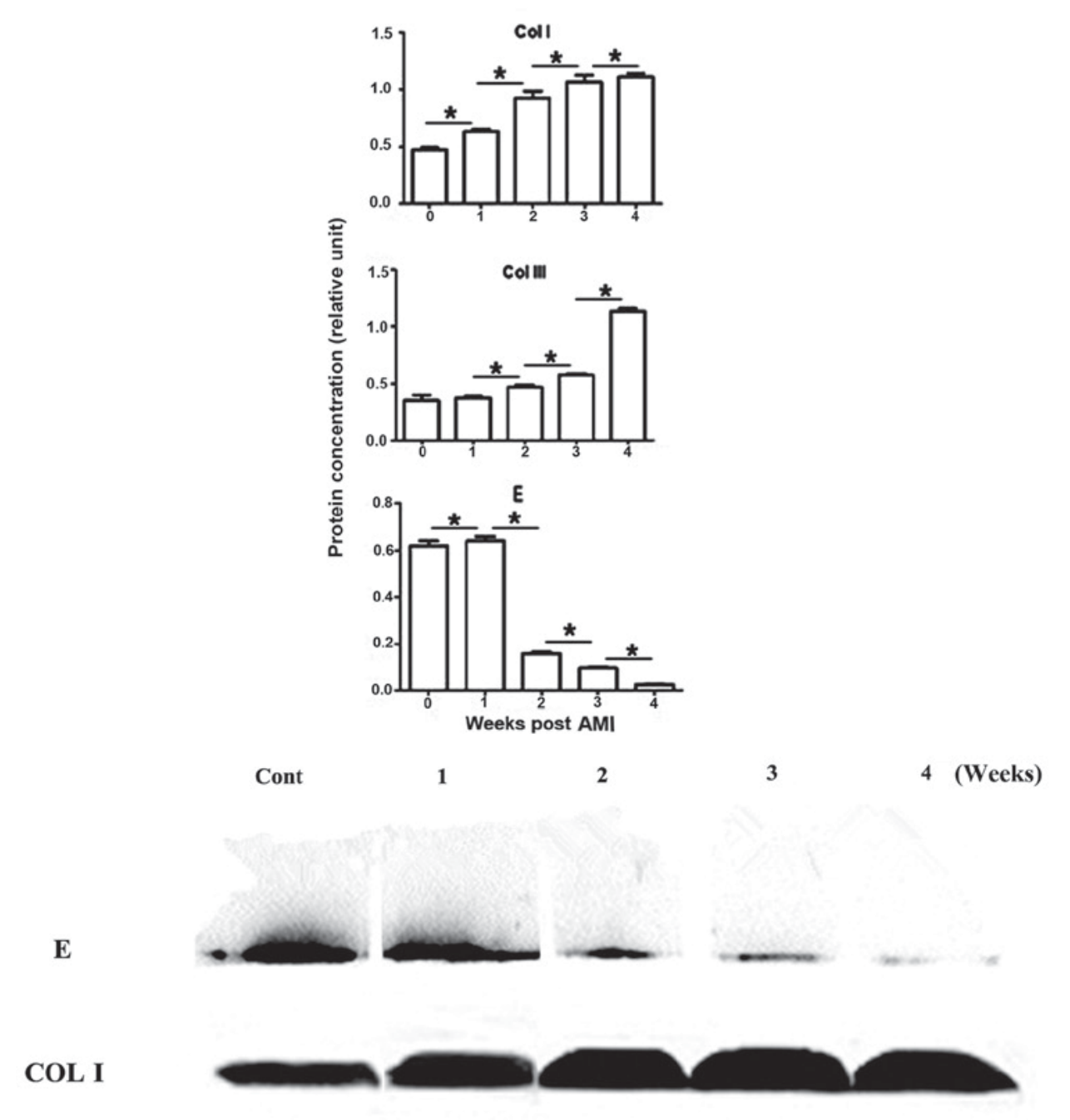

COL III

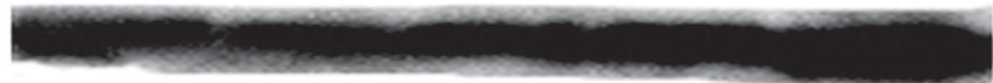

GADPH

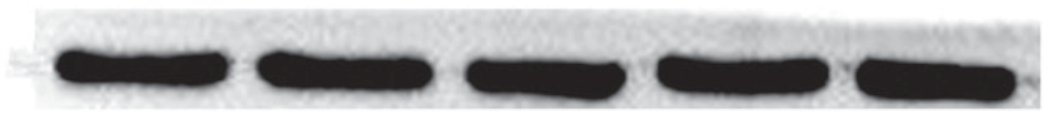

Figure 6. Protein expression levels of COL I, III and E fibres. Representative images and semi-quantitative analysis of protein expression levels of COL I, III and $\mathrm{E}$ fibres in the infarction zone of each group, detected by western blotting. " $\mathrm{P}<0.05$ as indicated. AMI, acute myocardial infarction; COL, collagen; E, elastin.

that adjustment of the content and structure of cardiac elastic fibre continues to occur with the altering course of the disease following myocardial infarction, indicating that elastic fibre takes part in the ventricular remodelling that follows myocardial infarction (16). In addition, elastic fibre markedly reduces in size and quantity in the maturation of scarring following AMI (17).

However, the dynamic alterations of collagen fibre and elastic fibre, and the alterations in ratio of one counterpart to the other in the context of AMI, remain to be elucidated and have not been reported to date, particularly regarding their association with cardiac function. The present study demonstrated that the content of collagen I fibre increased gradually. There was consistency between the upregulation of collagen I/III and the downregulation of cardiac function when comparing alterations in collagen with cardiac function. Reduction in cardiac function was inversely associated with the greatest induction of collagen following AMI. This finding is supported by the result from a previous study, which demonstrates that a novel collagen-derived matricryptin is generated post-MI, that mediates remodelling of the left ventricle (3).

Furthermore, the data demonstrated that elastic fibre decreased in the myocardial infarction zone gradually over four weeks AMI, which was consistent with reduced cardiac function over the period of AMI. The maximum reduction of elastic fibre was in the 2-3 week period, which was associated with the fastest reduction of cardiac function post AMI. According to Fung's Theory, elastin is the most 'linearly' elastic biosolid material, which is extensively distributed in 
animal tissues and organs. If a cylindrical specimen of elastin is prepared and subjected to an unaxial load in a tensile testing machine, the existence of an energy dissipation in the material is small (18). From the view of biomechanics, the increase or decrease of elastic fibres should not exert too much influence on heart function. Therefore, whether there is a causal association between the decrease of elastic fibres and the decline of cardiac function requires further research. The data from the present study is in accordance with previous studies, and suggested that increased elastic fragments in the area of myocardial infarction in rats altered the extracellular matrix of the infarcted zone during ventricular remodelling. Injection of elastic fragments into the AMI zone forms an organic extracellular matrix structure, and ultimately suppresses adverse modification in ventricular remodelling $(15,17)$. Finally, the ratio of elastic fibre and collagen increases, which may significantly improve the heart function, reduce scar area, and decrease ventricular dilatation $(19,20)$.

The results of the present study demonstrated that there was an association between the reduction of elastic fibre and induction of collagen I/III during AMI, and in particular, these alterations were consistent with impairment of cardiac function. However, the specific molecular mechanism underlying the process of pathological alteration following myocardial infarction, and the association with the presence of elastic fibre and collagen fibre, remains to be fully elucidated. The data suggested that decreased elasticity occurred in conjunction with increased 'hardness' in the formation of myocardial infarction scar tissue. Cardiac tissue flexibility or stiffness may be determined by the composition of elastic or collagen fibre during and/or post AMI (21). The data additionally suggested that inhibition of collagen and/or induction of elastic fibre may act as an effective approach to preserve or improve cardiac function during and/or post AMI.

Under various circumstances, animal gender may influence the research results. According to the author's previous study, there were differences in cells, tissues and their functions, between the different sexes, however there were almost no differences of the intercellular substance between males and females, including in collagen fibres and elastic fibres. Therefore, the heart of female rats only were selected as the research object of the present study (22).

In conclusion, the results of the present study demonstrated that in myocardial infarction, not only were cardiomyocytes damaged, but dynamic alterations in collagen and elastic fibers were also detected in the interstitium of the myocardium. Alterations in myocardial collagen and elastic fibers may also affect myocardial function, which has not yet attracted the attention of clinical diagnosis and treatment. Our future studies aim to improve and adjust the structural alterations in myocardial collagen and elastic fibers following myocardial injury, and make it more suitable for myocardial repair process.

\section{Acknowledgements}

The present study was supported by The Innovation Support Plan from Xinxiang Medical University (grant no. YJSCX201302Z) and The National Natural Science Fund Project (grant nos. 81400237 and 81570268), China. YY and
YG performed the experiment and interpreted the data; YY drafted the manuscript. SB interpreted the data and proof-read the manuscript, GZ designed the experiment and final proof-read the manuscript.

\section{References}

1. Guo J and Gao C: Significance of metabolic changes of myocardial collagens in rat myocardial infarction model during ventricular remodelling. Chin J of Clin Rehab 9: 56-57, 2005 (In Chinese).

2. Wang ZF, Wang NP, Harmouche S, Philip T, Pang XF, Bai F and Zhao ZQ: Retraction note to: Postconditioning promotes the cardiac repair through balancing collagen degradation and synthesis after myocardial infarction in rats. Basic Res Cardiol 110: 12, 2015.

3. Lu X: Physiological and pathological significance of myocardial interstitial networks. Basic Clin Med 12: 17-21, 1999(In Chinese).

4. Lindsey ML, Iyer RP, Zamilpa R, Yabluchanskiy A, DeLeon-Pennell KY, Hall ME, Kaplan A, Zouein FA, Bratton D, Flynn ER, et al: A novel collagen matricryptin reduces left ventricular dilation post-myocardial infarction by promoting scar formation and angiogenesis. J Am Coll Cardiol 66: 1364-1374, 2015.

5. Zuo H, Li C and Guo Z: Relationship between cardiac function and angiogenesis after rat myocardial infarction. Acta Anatomica Sinica 45: 35-40, 2014.

6. Guo Z: Current Cardiac Histology. People Health Publishing Company, Beijing, 2016.

7. Cai XH, Guo ZK, Mao HL and Qin C: Histologial architecture of reticular fibers and age-ralated changes of myocardial tissue in rats. Acta Anatomica Sinica 40: 127-129, 2009.

8. Kong CH, Lin XY, Woo CC, Wong HC, Lee CN, Richards AM, Sorokin VA: Characteristics of aortic wall extracellular matrix in patients with acute myocardial infarction: Itissue microarray detection of collagen I, collagen III and elastin levels. Interact Cardiovase Thorac Surg 16: 11-15, 2013.

9. Li G, Liu X and Tian L: A study on myocardial collagen synthesis and cardiac function in patients with anterior acute myocardial infarction without early successful reperfusion. J Postgrad Med 28: 27-29, 2005.

10. Serpooshan V, Zhao M, Metzler SA, Wei K, Shah PB, Wang A, Mahmoudi M, Malkovskiy AV, Rajadas J, Butte MJ, et al: The effect of bioengineered acellular collagen patch on cardiac remodeling and ventricular function post myocardial infarction. Biomaterials 34: 9048-9055, 2013.

11. Liu H, Wise SG, Rnjak-Kovacina J, Kaplan DL, Bilek MM, Weiss AS, Fei J and Bao S: Biocompatibility of silk-tropoelastin protein polymers. Biomaterials 35: 5138-5147, 2014.

12. Zhai X, Wu J, Guo Z and Wang L: Age-related changes of elastic fibers and elastic protein in rat ventricular myocardium. Acta Anatomica Sinica 40: 127-129, 2009.

13. Voorhees AP, DeLeon-Pennell KY, Ma Y, Halade GV, Yabluchanskiy A, Iyer RP, Flynn E, Cates CA, Lindsey ML and Han HC: Building a better infarct: Modulation of collagen cross-linking to increase infarct stiffness and reduce left ventricular dilation post-myocardial infarction. J Mol Cell Cardiol 85: 229-239, 2015.

14. Hervas A, Ruiz-Sauri A, de Dios E, Forteza MJ, Minana G, Nunez J, Gomez C, Bonanad C, Perez-Sole N, Gavara J, et al: Inhomogeneity of collagen organization within the fibrotic scar after myocardial infarction: Results in a swine model and in human samples. J Anat 228: 47-58, 2016.

15. Mizuno T, Mickle DA, Kiani CG and Li RK: Overexpression of elastin fragments in infarcted myocardium attenuates scar expansion and heart dysfunction. Am J Physiol Heart Circ Physiol 288: H2819-H2827, 2005.

16. Vander Donckt C, Van Herck JL, Schrijvers DM, Vanhoutte G, Verhoye M, Blockx I, Van Der Linden A, Bauters D, Lijnen HR, Sluimer JC, et al: Elastin fragmentation in atherosclerotic mice leads to intraplaque neovascularization, plaque rupture, myocardial infarction, stroke and sudden death. Eur Heart J 36: 1049-1058, 2015.

17. Li SH, Sun Z, Guo L, Han M, Wood MF, Ghosh N, Vitkin IA, Weisel RD and Li RK: Elastin overexpression by cell-based gene therapy preserves matrix and prevents cardiac dilation. J Cell Mol Med 16: 2429-2439, 2012. 
18. Fung YC: Mechanical properties of living tissue: Chapter 7 , Springer Verlag, New York, NY, pp242-245, 1993.

19. Lichtenauer M, Mildner M, Baumgartner A, Hasun M, Werba G, Beer L, Altmann P, Roth G, Gyöngyösi M, Podesser BK and Ankersmit HJ: Intravenous and intramyocardial injection of apoptotic white blood cell suspensions prevents ventricular remodelling by increasing elastin expression in cardiac scar tissue after myocardial infarction. Basic Res Cardiol 106 645-655, 2011.

20. Uchinaka A, Kawaguchi N, Hamada Y, Miyagawa S Saito A, Mori S, Sawa Y and Matsuura N: Transplantation of elastin-secreting myoblast sheets improves cardiac function in infarcted rat heart. Mol Cell Biochem 368: 203-214, 2012.
21. Liu Y, Xu Y, Wang Z, Wen D, Zhang W, Schmull S, Li H, Chen $Y$ and $X u e ~ S$ : Electrospun nanofibrous sheets of collagen/elastin/polycaprolactone improve cardiac repair after myocardial infarction. Am J Transl Res 8: 1678-1694, 2016.

22. Zhai XB, Wu JF, Guo ZK, et al: Aging changes of elastic fibers and collagen fibers in normal myocardium of rats. Acta Anatomica Sinica 44: 106-110, 2013.

(i) (9) This work is licensed under a Creative Commons

Attribution-NonCommercial-NoDerivatives 4.0 International (CC BY-NC-ND 4.0) License. 